\title{
A REVIEW OF UNCERTAINTY MEASUREMENT METHOD FOR OPTICAL DIMENSIONAL METROLOGY EQUIPMENT
}

\author{
M. P. CALVO-CORREA ${ }^{1}$, C. J. CORTÉS-RODRÍGUEZ ${ }^{1}$ \& JULIÁN R. CAMARGO L. ${ }^{2}$ \\ ${ }^{1}$ Mechanical Engineering Faculty, Universidad Nacional de Colombia, Bogotá, Colombia \\ ${ }^{2}$ Engineering Faculty, Universidad Distrital Francisco José de Caldas, Bogotá, Colombia
}

\begin{abstract}
Nowadays the manufacturing process is more efficient and faster than ever, creating the need for more sophisticated, and accurate dimensional measuring devices. In this context, the development of non-contact dimensional metrology equipment has helped in manufacturing advances. But what if the measure done for these devices isn't as exact as it needs. The determination and correction of the uncertainty are crucial for the accuracy of the measurements. This paper analyses the actual state of the determination of uncertainty for non-contact dimensional metrology equipment, especially for the focal variation devices. The researchers found that at present time there isn't much progress in the determination of uncertainty for non-contact dimensional metrology equipment and further investigations need to be done.

KEYWORDS: Focus Variation (FV), Metrological Dimensional Devices, Non-contract Equipment, Optical Dimensional Devices, Uncertainty
\end{abstract}

Received: Oct 06, 2020; Accepted: Oct 26, 2020; Published: Nov 28, 2020; Paper Id.: IJMPERDOCT202071

\section{INTRODUCTION}

Over time, lots of optical equipment have been developed allowing the measurement of pieces without the inconvenience that tactile MCM equipment could have [1].

Optical metrology is a non-contact technique. One of the advantages that provide is that does not leave a mark over the material at the moment of taking the measurement. It also allows us to do a complete piece measurement and not only a one-section measurement [2].

Optical metrology is the science that uses light to measure different variables and quantities. Using energy detectors that measure the light intensity of a beam of light that hits the surface [3].

Non-contact metrology has advanced rapidly in the last 30 years, essentially due to the advance of technology. Most of the manufacturing process has changed to optical technologies mainly for two factors. First, the advances in computational technology that allows us to save and process the information acquired. Secondly, the need for the most sophisticated manufacturing techniques, as a consequence of more volume of pieces manufactured and sold [4], [5].

One of the main purposes of manufacturing quality is to ensure that piece number one has the same quality as piece number one thousand. So what happens when the measurement equipment didn't detect the faulty piece? Or what happens if the tip of an MCM has suffered any damage like bending or other? How are these problems going to 
be avoided and how these problems could be solved?

Many of the difficulties that the MCM presents could be easily solved using non-contact equipment. But this type of equipment has its kinds of problems. Being the technology relatively new, these types of equipment have high levels of error and uncertainty in their measurements [6], [7].

Different studies have been done to try to determine, solve and measure the error and the uncertainty produced by different types of optical equipment, but further work has to be done to have a complete scheme of the subject. This article will describe different types of techniques used to measure the error and the uncertainty in different optical dimensional metrology equipment, and the authors will do a personal review of what should be expected in future works [8], [9].

Measurement techniques have changed in the last decade due to the evolution of technologies and equipment that have made the measures more accurate.

In 1989 the meter was defined as the distance between the North Pole and the equator through the longitudinal line that passes through Paris. In 1960 that definition changed and took a new value based on the wavelength of the emission spectrum of the ${ }^{38} \mathrm{KR}$. Later, in 1983, the General Conference on Weight and Measures (CGPM) decided to adopt the actual value of a meter based on the speed of light in a vacuum. These continuous changes in the metrology of measurements have made organizations think about how traceability should be measured. How much uncertainty affects assessments [6], [10], [11].

\section{OPTICAL DIMENSIONAL METROLOGY}

Optical dimensional devices have been an extraordinary tool for the development and the accuracy of measurement. These devices have considerable precision, and the measurements taken had many significant numbers. But one of its biggest problems is that not always these measurements are going to be the same. On some occasions testing the same piece can have different results if it was tested in different labs, or if it was tested in different optical devices. Even when these results are the same in many cases, this doesn't mean that it is correct, this only means that the test has repeatability [12], [13].

The repeatability allows us to verify if it is done in the same equipment, that the measurement had consistency, but this doesn't mean the value obtained in the test is correct [14], [15].

In addition to the previous description, optical equipment has some additional particularities that increase the uncertainty and provide some difficulties in the measurements done, like the edges, empty spaces of a piece, changes in the light levels, etc. [16], [17].

Some investigation centers like the PTB now are developing and founding investigations in dimensional optical metrology, especially in the determination of uncertainty for the measurement of pieces [18]. The group also develops equipment and measurement materials for the measurement of 3D roughness, as well as standards that contribute to this process [11], [19], [20].

\section{CMM vs OPTICAL UNCERTAINTY STUDIES}

One of the biggest advantages that the optical equipment has is the number of points measure and their locations are higher than the ones in the tactile equipment. Figure 1 shows how the error measurement increases due to the number of contact points [21]. 

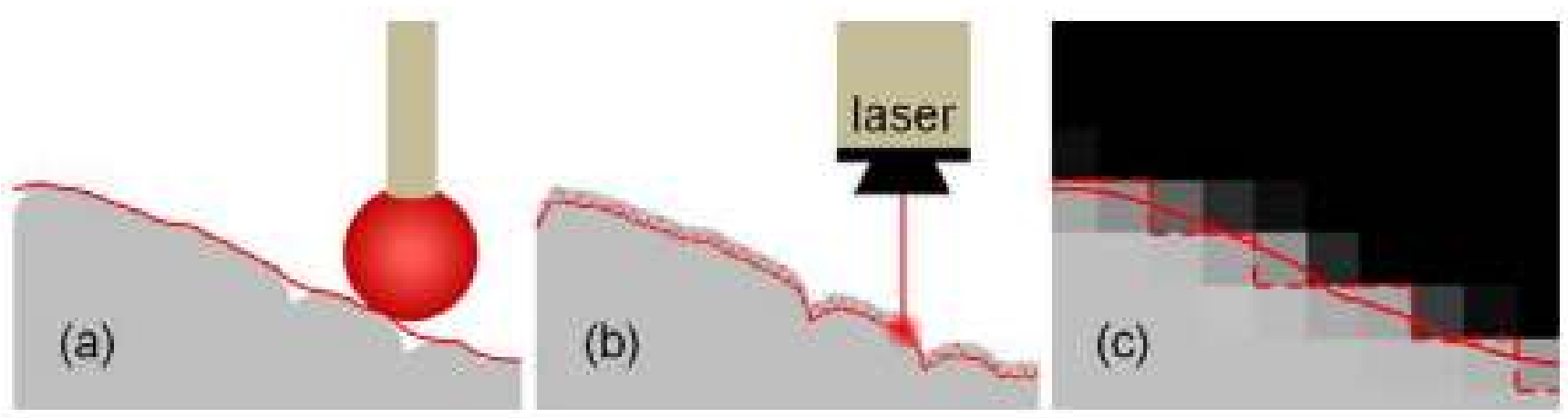

Figure 1: Schematic representation of measurement point of contact, a laser, and a CT scanning dimensional metrological equipment [21]

The last decade has developed different tools that have helped with the determination, description, and calculation of uncertainty and error on different types of dimensional metrology equipment. One of the most popular tools developed has been the reference spheres. These spheres have played an important role in the measurement of the accuracy of multisensor coordinate measuring machine (CMM) [15].

The calibration spheres should be round, hard, durable, small roughness, and non-magnetic [22].

These spheres have been developed to improve the accuracy of CMM and there exists a wide range of them. It doesn't mean that these spheres cannot be used in optical equipment. These are tools that have helped in the development of the measurement of the accuracy of uncertainty in optical devices [23], [24], [25].

In the case that these spheres want to be used in optical devices criteria such as geometrical, optical, and material characteristics must be taken into account for better results [26], [27].

Also, mathematical approaches have been done to determine and measure the error and uncertainty in the CMM equipment. Using statistical techniques like Monte Carlo and Neuronal network [12], [28], [29].

Seeing other's perspectives, like the one exposure for Sims, Isa et.al which expresses that most of the uncertainty work done for the CMM is already established, and major efforts should be done to determine the uncertainty in optical devices [30], [31].

An analysis of the Stereo-photogrammetry system using a gauge block and an interferometer laser to compare the results, helped the authors to determine the uncertainty using a traceable method resulting in the understanding of key influence factors of the uncertainty in the measurements using this equipment [30]. In this investigation, Monte Carlo's method allowed the researchers to determine the combined uncertainty for optical equipment demonstrating that one of the most relevant factors for the uncertainty in this equipment is the rotation of the camera and the distortion of parameters [32]. That investigation represented one of the first attempts of uncertainty analysis in a Stereo-photogrammetry system [30], [33], [34].

Monte Carlo Based methods provide a great solution due to the combined standard uncertainty for different systems, even complex ones [7]. Monte Carlo's method allows simulating different measurements in which the influence factors vary depending on the distribution [35].

Nowadays some other techniques have been proposed to determine the traceability, error, and uncertainty of optical 
metrology devices [36].

Authors as Hudson, Cline et. Al [37] proposed a crystal-based method to determine the traceability in the context of producing reference data for $\mathrm{x}$-ray wavelength using Bragg's Law.

In the same way, authors as Rodríguez-Sánchez, ÁngelaThompson [38] have described that one of the main reasons for uncertainty in XCT equipment is beam hardening, scattering, and blurring. They also described the complex relationship between the noise and the uncertainty in XCT equipment, making inefficiently detecting noise contribution alone [39].

Some other efforts have been done with Stereo Vision System equipment. Authors as Isa, sims, et.al, [40] have done volumetric error modeling to correct the error photogrammetric three-dimensional coordinate metrology. They presented a scheme to determine, measure, model, and correct the errors presented in the equipment using a laser interferometer while the stereo vision equipment was tracking the target points.

Some other technologies such as the focal variation have been developed to improve the metrological measurement. Focus variation is a non-contact technique that allows topographical measurements using optical at different depths and vertical scans [41], [42].

Focus variation works by looking for the focal position of an element, getting a map of the surface of the testing object [41]. This technique is not exempt from having the same problems of uncertainty, traceability, and error that some other optical dimensional metrology devices have presented [43].

Figure 2 shows the scheme of a Focus Variation device [44].

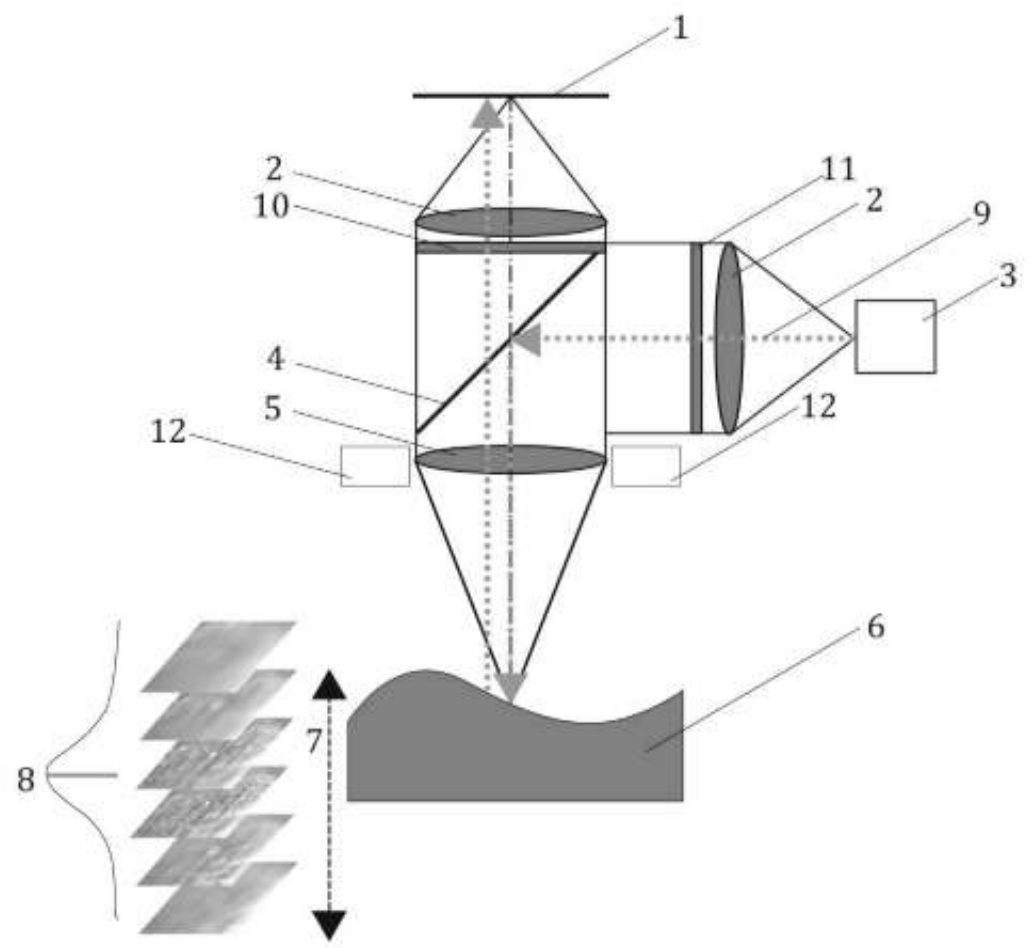

Figure 2: Focal variation device [44]

Some uncertainty sources identified for some researches [45] are the objective lenses, the light sources, the setting of the CCD sensor, the adjustment of the scan range, the lateral and vertical resolution, among others. This technology also 
presents a problem with the measurement angle, bigger than $87^{\circ}$ and slopes [46], [47].

Figure 3 shows how a Focal variation equipment measures samples with and angle higher than $90^{\circ}[45]$.
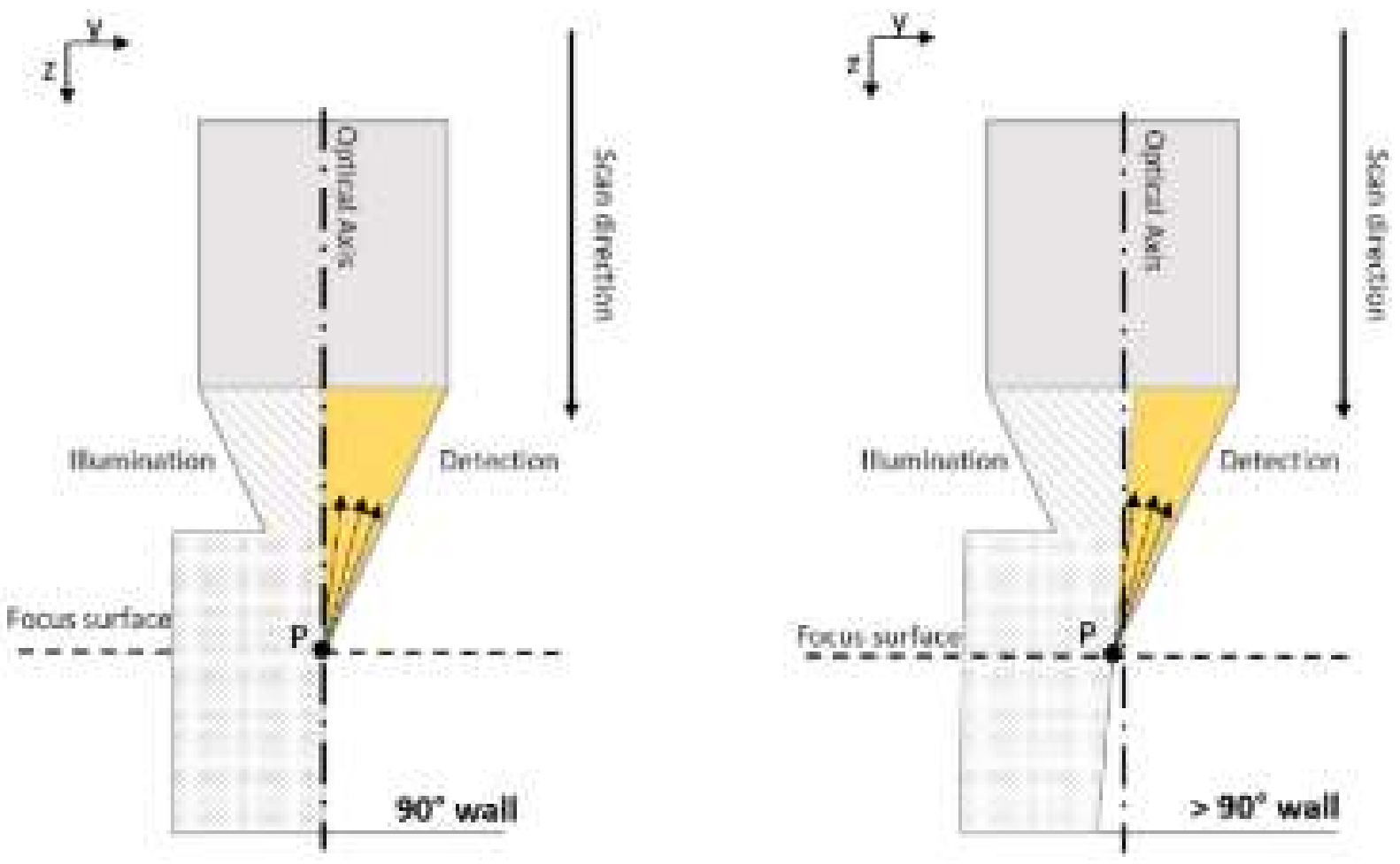

Figure 3: Scan detection in angles higher than $90^{\circ}[45]$

Nowadays, efforts have been done to create a guide to determine uncertainty, traceability, and error, but optical equipment hasn't advanced enough in this topic, making it difficult to have a certain method to make the data taken in this type of device accurate [48], [49], [50].

Some guides as the GUM [7] give a general description of what error and uncertainty are, and how they must be properly described and measured, but it doesn't have a specific description of uncertainty measurement for non-contact equipment.

On the other hand, the International Standard ISO 25178-606 [51] Geometrical product specification (GPS). Part: nominal characteristics of non-contact (Focus variation) instruments describes the metrological characteristics of a particular non-contact method for measuring surface texture. This standard used together with the GUM can provide a tool to create a scheme to determine, measure, detect, model, and correct the uncertainty in focal variation devices.

By the time of the writing of this article, the authors didn't find literature about traceability in focal variation devices.

\section{CONCLUSIONS}

This paper presents a general view of the actual state of the art of the uncertainty measurement method developed of optical 
or non-contact, especially for focal variation equipment. In general contact dimensional metrology devices, CMM has been widely studied, and several studies have been developed to determine the uncertainty for these devices. On the other hand, optical dimensional metrology devices have been left behind in the same topic, more studies need to be done about this subject. Using the GUM as a guide and using new statistical methods as Baye's approaches or Monte Carlo, or also using new data acquisition as neural networks can be useful in the approach to the determination and correction of uncertainty in non-contact devices especially focal variation.

\section{ACKNOWLEDGMENTS}

The authors would like to thank the Universidad Nacional de Colombia and to the GIBM - UNCB research group for supporting the development of this project.

\section{REFERENCES}

1. H. Imai, "Expanding needs for metrological traceability and measurement uncertainty," Meas. J. Int. Meas. Confed., vol. 46, no. 8, pp. 2942-2945, 2013.

2. T. Yoshizawa, Handbook of optical metrology: Principles and applications, second edition, 3rd ed. Yokohma, Japan: CRC Press. Taylor and Francis Group, 2015.

3. Rajpal S. Sirohi, Introduction to optical metrology, vol. 1, no. 1. Taylor and Francis Group, 2016.

4. “Applications of three-dimensional surface metrology,” Three Dimens. Surf. Topogr., pp. 175-213, 2000.

5. K. Harding, Handbook of optical dimensional metrology. CRC Press. Taylor and Francis Group, 2016.

6. C. E. de Metrología, "Guía para la Expresión de la Incertidumbre de Medida," 2008.

7. J. 101:2008 and Evaluation, "Evaluation of measurement data - Supplement 1 to the 'Guide to the expression of uncertainty in measurement' - Propagation of distributions using a Monte Carlo method," JCGM 1012008 Eval., vol. 1, no. 1, p. 90, Sep. 2008 .

8. M. Eifler et al., "Comparison of material measures for areal surface topography measuring instrument calibration," Surf. Topogr. Metrol. Prop., vol. 8, no. 2, p. 025019, 2020.

9. L. Todhunter, R. Leach, and F. Blateyron, "Mathematical approach to the validation of surface texture filtration software," Proc. 20th Int. Conf. Eur. Soc. Precis. Eng. Nanotechnology, EUSPEN 2020, no. October, pp. 353-356, 2020.

10. Saudi and O. Standards, "KINGDOM OF SAUDI ARABIA GUIDE TO THE EXPRESSION OF UNCERTAINTY IN MEASUREMENT," 2006

11. Joint Committee For Guides In Metrology, "Evaluation of measurement data - Guide to the expression of uncertainty in measurement," 2008.

12. F. J. Raya de Blas, "Trazabilidad de la medición por coordenadas aplicando simulación estadística y redes neuronales," 2015.

13. C. J. L. Pérez, "Surface roughness modelling considering uncertainty in measurements, " Int. J. Prod. Res., vol. 40, no. 10, pp. 2245-2268, 2002. 
14. H. Villarraga-Gómez, J. D. Thousand, S. T. Smith, H. Villarraga-Gómez, J. D. Thousand, and S. T. Smith, "Empirical approaches to uncertainty analysis of X-ray computed tomography measurements: A review with examples," Precis. Eng., vol. 64, pp. 249-268, 2020.

15. C. P. Keferstein et al., "Universal high precision reference spheres for multisensor coordinate measuring machines," CIRP Ann. - Manuf. Technol., vol. 61, no. 1, pp. 487-490, 2012.

16. S. J. Harrison and R. H. Dieck, "Measurement errors and uncertainty," in The Engineering Handbook, Second Edition, 2004, pp. 152-1-152-9.

17. P. Ii, "Instruments and measurement techniques of three-dimensional surface topography," Three Dimens. Surf. Topogr., pp. 19-94, 2000.

18. W. Group, “3D Roughness Metrology,” 2020.

19. L. D. A. Montero, P. A. F. Mosquera, and O. David Florez Cediel, "Forecasting and development of a wind speed emulator based on computational intelligence techniques, " in 2015 IEEE Workshop on Power Electronics and Power Quality Applications, PEPQA 2015 - Proceedings, 2015.

20. C. Barragan, C. A. Bautista, and O. Florez, "Evaluation of the energetic and operational efficiency in active power filters using fuzzy logic control for microgrid applications, " in 2015 IEEE Workshop on Power Electronics and Power Quality Applications, PEPQA 2015 - Proceedings, 2015.

21. S. Carmignato and E. Savio, “Traceable volume measurements using coordinate measuring systems," CIRP Ann. - Manuf. Technol., vol. 60, no. 1, pp. 519-522, 2011.

22. Weckenmann et al., "Multisensor data fusion in dimensional metrology,” CIRP Ann. - Manuf. Technol., vol. 58, no. 2, pp. 701-721, 2009.

23. Küng, F. Meli, and R. Thalmann, “Ultraprecision micro-CMM using a low force 3D touch probe, ” in Measurement Science and Technology, 2007, vol. 18, no. 2, pp. 319-327.

24. S. Carmignato, L. De Chiffre, H. Bosse, R. K. Leach, A. Balsamo, and W. T. Estler, "Dimensional artefacts to achieve metrological traceability in advanced manufacturing," CIRP Ann., pp. 1-24, 2020.

25. J. D. Claverley and R. K. Leach, "A review of the existing performance verification infrastructure for microCMMs, " Precis. Eng., vol. 39, pp. 1-15, 2015.

26. X. Zhang et al., "Correlation approach for quality assurance of additive manufactured parts based on optical metrology, ”. Manuf. Process., vol. 53, pp. 310-317, 2020.

27. S. Shi, R. Kuschmierz, G. Zhang, J. Lin, J. Czarske, and J. Qu, "Modeling, quantification, and mitigation of uncertainty propagation in two-step roundness measurements,” Meas. J. Int. Meas. Confed., vol. 155, 2020.

28. Štrbac, B. Ačko, S. Havrlišan, I. Matin, S. Savkovicá, and H. Hadžistevicá, "Investigation of the effect of temperature and other significant factors on systematic error and measurement uncertainty in CMM measurements by applying design of experiments, " 2020.

29. F. J. Pontes et al., "Optimization of Radial Basis Function neural network employed for prediction of surface 
roughness in hard turning process using Taguchi's orthogonal arrays, ” Expert Syst. Appl., vol. 39, no. 9, pp. 77767787, 2012.

30. Sims-Waterhouse, M. Isa, S. Piano, and R. Leach, "Uncertainty model for a traceable stereo-photogrammetry system," Precis. Eng., vol. 63, pp. 1-9, 2020.

31. W. Dewulf, K. Kiekens, Y. Tan, F. Welkenhuyzen, and J. P. Kruth, "Uncertainty determination and quantification for dimensional measurements with industrial computed tomography," CIRP Ann. - Manuf. Technol., vol. 62, no. 1, pp. 535-538, 2013.

32. G. Moona, V. Kumar, M. Jewariya, R. Sharma, and H. Kumar, "Measurement Uncertainty Evaluation Using Monte Carlo Simulation for Newly Established Line Scale Calibration Facility at CSIR-NPLI, ” MAPANMapan - J. Metrol. Soc. India, vol. 34, no. 3, pp. 325-331, 2019.

33. F. Lavecchia, M. G. Guerra, and L. M. Galantucci, "Performance verification of a photogrammetric scanning system for micro-parts using a three-dimensional artifact: adjustment and calibration," Int. J. Adv. Manuf. Technol., vol. 96, no. 9-12, pp. 4267-4279, Jun. 2018.

34. P. Sun, N.-G. Lu, M.-L. Dong, B.-X. Yan, and J. Wang, "Simultaneous All-Parameters Calibration and Assessment of a Stereo Camera Pair Using a Scale Bar."

35. J. Stadek and A. Ga̧ska, "Evaluation of coordinate measurement uncertainty with use of virtual machine model based on Monte Carlo method," Meas. J. Int. Meas. Confed., vol. 45, no. 6, pp. 1564-1575, 2012.

36. L. Tonietto, L. Gonzaga, M. R. Veronez, C. de S. Kazmierczak, D. C. M. Arnold, and C. A. da Costa, "New Method for Evaluating Surface Roughness Parameters Acquired by Laser Scanning," Sci. Rep., vol. 9, no. 1, pp. 1-16, 2019.

37. L. T. Hudson, J. P. Cline, A. Henins, M. H. Mendenhall, and C. I. Szabo, "Contemporary x-ray wavelength metrology and traceability," Radiat. Phys. Chem., vol. 167, p. 108392, 2020.

38. A. Rodríguez-Sánchez, A. Thompson, L. Körner, N. Brierley, and R. Leach, "Review of the influence of noise in Xray computed tomography measurement uncertainty, ” Precision Engineering, vol. 66. pp. 382-391, 2020.

39. J. J. Lifton and T. Liu, "Evaluation of the standard measurement uncertainty due to the ISO50 surface determination method for dimensional computed tomography," Precis. Eng., vol. 61, pp. 82-92, 2020.

40. M. A. Isa, D. Sims-Waterhouse, S. Piano, and R. Leach, "Volumetric error modelling of a stereo vision system for error correction in photogrammetric three-dimensional coordinate metrology, ” Precis. Eng., vol. 64, pp. 188-199, 2020.

41. R. Leach, Optical Measurement of Surface Topography. 2011.

42. "Focus Variation| Form \& roughness with one system | Alicona." [Online]. Available: https://www.alicona.com/en/our-technology/focus-variation/. [Accessed: 25-Aug-2020].

43. R. Danzl, F. Helmli, and S. Scherer, "Focus variation - A robust technology for high resolution optical 3D surface metrology," Stroj. Vestnik/Journal Mech. Eng., 2011. 
44. Optical Measurement of Surface Topography. 2011.

45. K. Zangl, B. Alicona, R. Danzl, B. Alicona, and F. Helmli, "Vertical Focus Probing and the use in Dimensional Metrology," Pre print, no. April, pp. 1-8, 2019.

46. B. Muralikrishnan, J. A. Stone, and J. R. Stoup, "Fiber deflection probe for small hole metrology," Precis. Eng., 2006.

47. "Infinite Focus|Dimensional accuracy \& roughness | Alicona." [Online]. Available: https://www.alicona.com/en/products/infinitefocus/. [Accessed: 31-Aug-2020].

48. Zoraima and A. Salazar, superficies : forma y rugosidad 3D con método óptico de variación focal. 2018.

49. B. Daemi, P. Ekberg, and L. Mattsson, "Lateral performance evaluation of laser micromachining by high precision optical metrology and image analysis," Precis. Eng., vol. 50, pp. 8-19, 2017.

50. Liu, E. Lu, H. Yi, M. Wang, and P. Ao, “A new surface roughness measurement method based on a color distribution statistical matrix,” Meas. J. Int. Meas. Confed., vol. 103, no. February, pp. 165-178, 2017.

51. “ISO 25178-606.pdf,” Switzerland, 2015. 

